\title{
Nanoscale fracture studies using the scanning force microscope
}

\author{
Bettina Baumeister ${ }^{\mathrm{a})}$ and Thomas A. Jung \\ Laboratory for Micro- and Nanotechnology, Paul-Scherrer-Institute, 5232 Villigen PSI, Switzerland \\ Ernst Meyer \\ Institute of Physics, Condensed Matter Department, University of Basel, 4056 Basel, Switzerland
}

(Received 6 September 2000; accepted for publication 22 February 2001)

\begin{abstract}
We present a variety of experiments concerning friction and fracture mechanisms using two-dimensional arrays of microfabricated nanotowers as templates. The scanning force microscope tip is used as a tool to apply well-defined forces to the surface of the patterned substrate. Force statistic measurements reveal information about the forces involved in the fracture process and the probability of fracture of selected towers. These methods are discussed in the context of nanometer-scale mechanisms. Using optimized parameters, a controlled removal of individual nanotowers and the ability to "write" predefined patterns on a nanometer scale can be achieved.

(C) 2001 American Institute of Physics. [DOI: 10.1063/1.1367297]
\end{abstract}

In the past few years many efforts have been made towards controlled surface modification using scanning probe microscopy techniques. Writing patterns in polymers, ${ }^{1}$ the modification of semiconductor surfaces, e.g., producing lines ${ }^{2,3}$ and dots, ${ }^{4,5}$ and the positioning of atoms ${ }^{6}$ and molecules, ${ }^{7}$ nanocrystals, ${ }^{8}$ and particles ${ }^{9}$ have been performed successfully. Nanotribological investigations with simultaneous measurements of topography and friction forces can be performed using the scanning force microscope (SFM). ${ }^{10}$ The forces involved in the sliding or rolling of nanocrystals ${ }^{8}$ or carbon nanotubes ${ }^{11}$ on substrates have been examined.

Here, we present a method to perform nanoscale experiments using the scanning force microscope: using lithographically produced nanostructures, fracture and friction are explored at small dimensions.

We used a Topometrix Explorer SFM in contact mode at room temperature. The silicon-nitride tips (Microlevers by Park Scientific Instruments, type A) were calibrated by the method described by Hutter and Bechhoefer ${ }^{12}$ obtaining a spring constant of $0.045 \mathrm{~N} / \mathrm{m}$. All experiments were performed in bi-distilled water with samples consisting of square $\mathrm{SiO}_{2}$-nanotower arrays on a silicon substrate produced by laser interference lithography. ${ }^{13}$ The lattice constant is $680 \mathrm{~nm}$ and the height of the towers is $120 \mathrm{~nm}$ [Fig. 1(a)]. The image in Fig. 1(a) has been achieved by scanning in contact mode with a normal force of $4 \mathrm{nN}$ ("imaging mode"). Moving the SFM tip laterally across one selected tower with an increased normal force results in fracture and removal of this tower [see the center nanotower in Figs. 1(a) and 1(b)]. In a detailed series of experiments, all relevant parameters have been identified and a variety of surface modifications have been observed. Fracture can be achieved by two different modes: In the first mode, the normal force $F_{N}$ is set to a fixed value of up to $25 \mathrm{nN}$ ["static modification mode," Fig. 1(c)]; in the second mode the normal force is doubled with respect to the imaging mode and cantilever oscillations with frequencies of $1.75 \mathrm{kHz}$ and amplitudes of $25 \mathrm{~nm}$ exert periodic forces in the contact area of the canti-

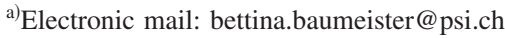

lever tip ("dynamic modification mode"). The involved static and dynamic force magnitudes are the main parameters to control the modification process. The frequency of force modulation was not observed to be of critical importance in the range of 1-3 kHz. In both modification modes the structure breaks at the bottom. The roughness of the residual surface left after removal of the tower is $<3 \mathrm{~nm}$. This indicates that the fracture occurs at the $\mathrm{SiO}_{2} / \mathrm{Si}$ interface at the bottom of the towers [Fig. 1(c)].

In repeated experiments the statistics of nanotower breaking in the static modification mode has been analyzed. Scanning across $10 \times 10 \mu \mathrm{m}^{2}$ with an increased force removes a certain fraction of towers; this fraction depends on the force $F$ that is applied to the towers. From the subsequent (a)

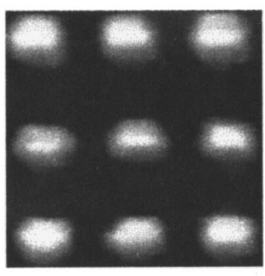

(c)

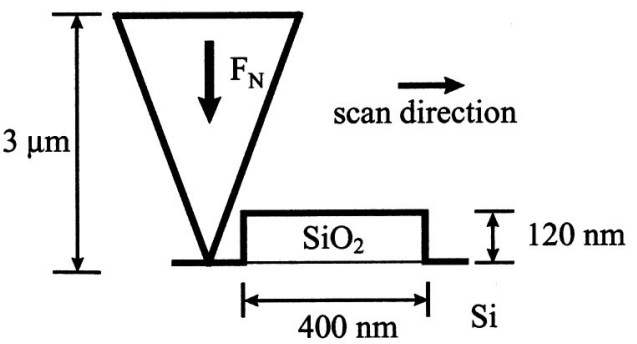

FIG. 1. Selective removal of $\mathrm{SiO}_{2}$ nanotowers using scanning force microscopy. (a) $\mathrm{SiO}_{2}$ nanotowers with a lattice constant of $680 \mathrm{~nm}$ and a height of $120 \mathrm{~nm}$ on a Si substrate produced by laser interference lithography. (b) Moving the SFM tip across the center tower in (a) with a normal force $F_{N}$ of $20 \mathrm{nN}$ results in fracture and displacement. Fracture can be achieved by two different techniques: increasing the normal force (static modification mode) or increasing the normal force and exciting cantilever oscillations additionally (dynamic modification mode). In both cases, the structure breaks at the bottom. The roughness of the residual surface left after removal of the tower is $<3 \mathrm{~nm}$. This indicates that the fracture occurs at the $\mathrm{SiO}_{2} / \mathrm{Si}$ interface at the bottom of the towers. (c) Diagram of the static modification process. The normal force $F_{N}$ is increased with respect to the imaging mode to a fixed value of up to $25 \mathrm{nN}$. The $\mathrm{SiO}_{2} / \mathrm{Si}$ interface is denoted by the thin line. 
(a)

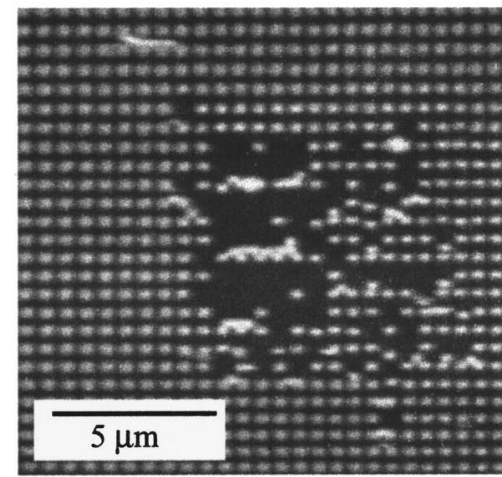

(b)

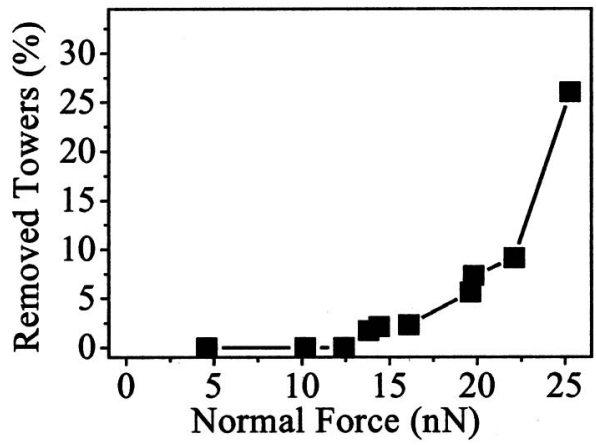

(c)

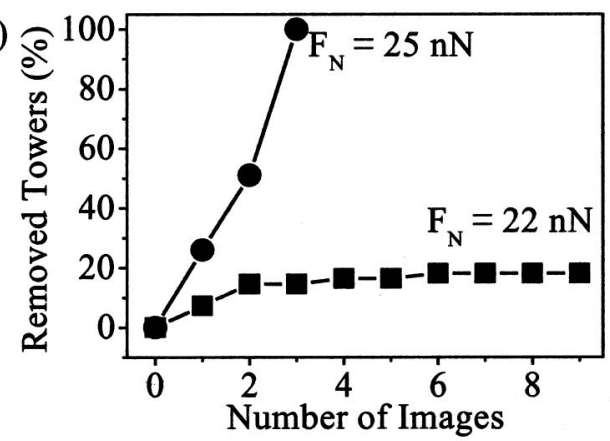

FIG. 2. Force-dependent statistics of nanotower removal. (a) Scanning across $10 \times 10 \mu \mathrm{m}^{2}$ in static modification mode removes a certain fraction of towers. From the subsequent overview taken in imaging mode this fraction can be determined. (b) The percentage of removed towers is plotted for a series of such experiments with varied modification force $(5-25 \mathrm{nN})$. No nanotowers have been removed below the observed threshold loading of 13 $\mathrm{nN}$. (c) With repeated static modification mode scanning a monotonously increasing number of nanotowers is removed. Applying $22 \mathrm{nN}$, the number of removed towers reaches a value of $18 \%$ asymptotically. Using only $3 \mathrm{nN}$ more, the surface is completely cleaved after three scans.

overview taken in the imaging mode [Fig. 2(a)], we observe the fraction of the towers that was removed in the previous static modification mode scan. Tower fracture originates from the force $F$ superimposed from lateral and normal forces while scanning the tip across the sample. The coefficient of friction for the investigated system was experimentally determined to be 0.22 . From geometric calculations we estimate that the absolute value of the force $F$ acting on the towers is in the same order of magnitude as the normal force $F_{N}$. The applied force $F$ is proportional to the measured normal force $F_{N}$ with given frictional and geometrical parameters. Our estimate is experimentally confirmed by measurements of the lateral forces when breaking some towers. Because of this proportionality $\left(F \sim F_{N}\right)$ we use the normal force $F_{N}$ which is directly accessible to describe the results of our experiments.

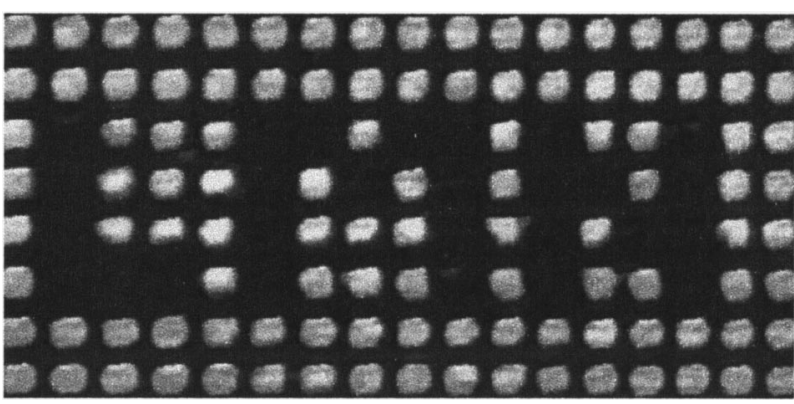

FIG. 3. Nanopattern generated using the described dynamic modification method after detailed optimization of all relevant parameters. Individual nanotowers have been directly accessed and removed. The achieved degree of control demonstrates the potential of the presented weak link concept for technological exploitation.

In Fig. 2(b), the percentage of removed towers is plotted for a series of such experiments with varied modification force $(5-25 \mathrm{nN})$. No nanotowers have been removed below the observed threshold loading of $13 \mathrm{nN}$. Repeated scanning in static modification mode increases the number of removed towers monotonously [Fig. 2(c)]. Applying a force of $22 \mathrm{nN}$ to the surface, the fraction of removed towers reaches a value of $18 \%$ asymptotically. Using $25 \mathrm{nN}$, the surface is completely cleaved after only three scans. Thus, the force difference $\Delta F$ of only $3 \mathrm{nN}$ determines whether $18 \%$ or $100 \%$ of the towers are cleaved. Assuming that this $\Delta F$ corresponds to a deviation in tower thickness solely, this thickness deviation can be calculated to $35 \%$. From SFM analysis of the nanotowers we determine the thickness deviation of the individual nanotowers to be below $10 \%$. Therefore, the explanation for the force difference $\Delta F$ goes beyond the observed size or width fluctuations, but originates from internal structural variations. Possible mechanisms include the weakening of the interface by strain and chemical agents (liquid environment and etching during manufacturing). Recently, dislocation defects at the $\mathrm{SiO}_{2} / \mathrm{Si}$ interface in silicon-on-insulator (SOI) wafers have been observed to induce strain. ${ }^{14}$ Our considerations are also supported by investigations of chemical interactions with intrusive water at fracture interfaces in mica. ${ }^{15}$ These explorations show that a reduced interface energy in water results in a decreasing force required for initiation of brittle fracture. ${ }^{15}$ Using the described microfabricated nanotowers with well-defined material interfaces, SFM measurements of normal and lateral forces enable conclusive experiments about fracture in response to applied forces. The initiation and progress of fracture on the nanometer scale and down to atomic or molecular size are also of significant importance for macroscopic behavior.

Using a combination of imaging mode and dynamic modification mode, a controlled nanotower removal technique is achieved. After optimization of all relevant parameters in dynamic modification mode, individual nanotowers can be directly accessed and removed. Following each sequence of modification experiments, the site of removed towers and the progressively evolving pattern is imaged. Using this technique nanometer scale patterns can be generated. As an example of a complex pattern, we show the logo of our laboratory in Fig. 3. These results suggest the feasibility of the write once, read many times (WORM) type of data storage when using nanotower arrays with the stressed 
$\mathrm{SiO}_{2} / \mathrm{Si}$ interface as a well-defined "weak link" for mechanical removal. A variety of methods (e.g., masked x-ray lithography) allows the production of high-densitiy nanotower arrays for increased storage densities.

To conclude, we have presented investigations on fracture of nanotowers with an SFM tip. Force statistics reveal a very sharp threshold force for fracture initiation. Combining imaging and dynamic modification modes, the controlled removal of selected nanotowers has been achieved. Using this technique complex nanopatterns have been generated. From the observed qualitative and quantitative behavior reported here, we can foresee a number of experiments to elaborate tribological concepts on the nanometer scale. Most exciting is the ability to apply well-defined forces to wellcharacterized nanometer-sized structures with varying geometrical and structural parameters. The transition from the elastic and plastic behavior at large dimensions down to the atomic and molecular scale provides valuable insight into size effects as observed for electronic and optical properties. ${ }^{16,17}$ Possibly the closest mechanical analogue is the increase of hardness at small loads as has been observed as a result of surface tensions and strains with nanoindenter experimentation and simulation. ${ }^{18,19}$ Finally, the improved understanding of fracture on the nanometer scale may lead to concepts of data storage using engineered mechanical weak links within nanometer-scale structures.

The authors thank Loris Scandella for providing the nanostructures and for valuable conversations and express their thanks to Louis Tiefenauer for interesting and helpful discussions. Financial support from the Swiss National Science Foundation is gratefully acknowledged.

${ }^{1}$ T. A. Jung, A. Moser, H. J. Hug, D. Brodbeck, R. Hofer, H. R. Hidber, and U. D. Schwarz, Ultramicroscopy 42-44, 1446 (1992).

${ }^{2}$ C. K. Hyon, S. C. Choi, S. W. Hwang, D. Ahn, Y. Kim, and E. K. Kim, Appl. Phys. Lett. 75, 292 (1999).

${ }^{3}$ R. Magno and B. R. Bennett, Appl. Phys. Lett. 70, 1855 (1997).

${ }^{4}$ M. Wendel, S. Kühn, H. Lorenz, J. P. Kotthaus, and M. Holland, Appl. Phys. Lett. 65, 1775 (1994).

${ }^{5}$ J. Cortes Rosa, M. Wendel, H. Lorenz, J. P. Kotthaus, M. Thomas, and H. Kroemer, Appl. Phys. Lett. 73, 2684 (1998).

${ }^{6}$ D. M. Eigler and E. K. Schweizer, Nature (London) 344, 524 (1990).

${ }^{7}$ T. A. Jung, R. R. Schlittler, J. K. Gimzewski, H. Tang, and C. Joachim, Science 271, 181 (1996).

${ }^{8}$ P. E. Sheehan and C. M. Lieber, Science 272, 1158 (1996).

${ }^{9}$ T. Junno, K. Deppert, L. Montelius, and L. Samuelson, Appl. Phys. Lett. 66, 3627 (1995).

${ }^{10}$ R. Lüthi, E. Meyer, H. Haefke, L. Howald, W. Gutmannsbauer, and H.-J. Güntherodt, Science 266, 1979 (1994).

${ }^{11}$ M. R. Falvo, R. M. Taylor II, A. Helser, V. Chi, F. P. Brooks, Jr., S. Washburn, and R. Superfine, Nature (London) 397, 236 (1999).

${ }^{12}$ J. L. Hutter and J. Bechhoefer, Rev. Sci. Instrum. 64, 1868 (1993).

${ }^{13}$ M. Schildenberger, Y. Bonetti, M. Aeschlimann, L. Scandella, J. Gobrecht, and R. Prins, Catal. Lett. 56, 1 (1998).

${ }^{14}$ P. Sutter, M. G. Lagally, Phys. Rev. Lett. 82, 1490 (1999).

${ }^{15}$ B. Lawn, in Fundamentals of Friction: Macroscopic and Microscopic Processes, edited by I. L. Singer and H. M. Pollock (Kluwer Academic, Dordrecht, Netherlands, 1992), p. 137.

${ }^{16}$ F. J. Himpsel, T. A. Jung, and P. F. Seidler, IBM J. Res. Dev. 42, 33 (1998).

${ }^{17}$ M. V. Rama Krishna and R. A. Friesner, Phys. Rev. Lett. 67, 629 (1991).

${ }^{18}$ R. Komanduri, N. Chandrasekraran, and L. M. Raff, Phys. Rev. B 61, 14007 (2000).

${ }^{19}$ W. C. Oliver, R. Hutchings, and J. B. Pethica, in Microindentation Techniques in Materials Science and Engineering, edited by P. J. Blau and B. R. Lawn (International Metallographic Society, Philadelphia, PA, 1985), p. 91. 